\title{
Study On High Speed Driving Stability Of Tractor-Semitrailer
}

\author{
Chaoyi Wei ${ }^{1, a^{*}}$, Qunzhang Liang ${ }^{1, b}$, Zhenhui Chen $^{1, \mathrm{c}}$, Guosen Xue $\mathrm{e}^{1, \mathrm{~d}}$ \\ ${ }^{1}$ College of Mechanical Engineering, Guangxi University, Nanning, Guangxi Province, China \\ a gxdavid@163.com, ${ }^{b}$ qzgxdx@163.com, ${ }^{c}$ weipan1317@163.com, ${ }^{d}$ xgsufo@163.com
}

\begin{abstract}
Keywords: Tractor-Semitrailer; The High-speed Shimmy; Sliding Mode Variable Structure; Combined Simulation

Abstract. Because of its own characteristics and the complexities of driving conditions, Tractor-Semitrailer easily produce shimmy when driving at the high speed. In order to effectively control the high-speed shimmy of Tractor-Semitrailer, differential braking control strategy based on sliding mode variable structure control theory is proposed, and the Corresponding controller is designed. Then, the Simulink controller model and the TruckSim Tractor-Semitrailer vehicle dynamic model are respectively set up, then carried out co-simulation to compare controlled and uncontrolled Tractor-Semitrailer' driving state at high speed. The results show that the proposed control strategy can effectively reduce yaw velocity and roll angle produced by shimmy at high speed driving, so as to achieve the purposes that controlling the high-speed shimmy and improving the stability of Tractor-Semitrailer traveling at high speed .
\end{abstract}

\section{Introduction}

The semi-trailer train has the characteristics of high efficiency in transportation, convenient trailer transportation, low cost and so on. It has become the main force of highway transportation, especially in the middle-long distance highway transportation ${ }^{[1]}$. But due to its large size, high center of mass, large and changeable mass of payload and the intercoupling between the tractor and the trailer, it is very easy to occur accident of the rollover, shimmy and folding, resulting in huge losses ${ }^{[2-3]}$. According to statistics, the trailer's shimmy is prone to happen only after the vehicle's accident of rollover ${ }^{[4]}$. At the same time, because of the characteristics of the semi-trailer train itself, when the traffic accident occurs, it is often a serious accident which brings a particularly significant loss to people's life and property. Therefore, it is very urgent to analyze the mechanism of the high-speed shimmy of semi-trailer train and to study the method and means to reduce its high-speed shimmy to improve its stability and safety of high speed driving.

As the damping ratio of the vehicle system decreases with the increasing of vehicle speed ${ }^{[5]}$, once the system damping ratio is reduced to a certain degree, the lateral shimmy of the vehicle is generated under the interference of the external force. The structural parameters of the semi-trailer train are directly related to the damping ratio of the system, so the damping ratio of the system can be improved by changing the structural parameters of the vehicle. However, some parameters like the quality of the semi trailer, the front and rear position of the center of mass and the height of the center of mass will change with loading value and loading position changing. So the method of improving the damping ratio by changing structural parameters of vehicle is influenced by many factors. In the case of not changing the structural parameters of the vehicle, through the control method of differential braking based on sliding mode variable structure control is carried out to improve and enhance the lateral stability of the high-speed driving. This method is more simple and flexible.

\section{Establishment of Differential Braking System}

The use of established system of differential brake can monitor the yaw states of the tractor and the trailer in real time and then carry out differential brake control for wheels of the semi-trailer train in necessary. The differential brake control strategy is divided into two steps, the first step is to judge the shimmy orientation of vehicle based on the orientation of the tractor's yaw angle rate outputted from TruckSim vehicle model. The second step, differential braking is implemented. The second step, differential braking is implemented. According to yaw orientation and the situation of yaw changing, 
different braking forces are applied to different wheels to obtain the desired yaw moment and reduce the angle between the trailer and tractor. It also avoids due to the yaw too large to lead to dangerous folding occurred.

The differential braking strategy of the vehicle is shown in Table 1 , where $\varphi_{1}$ is defined as tractor's yaw angle, $\varphi_{2}$ is defined as trailer's yaw angle, $\varphi_{1}>0$ is defined as the left-yaw orientation of the vehicle, and $\varphi_{1}<0$ is defined as the right-yaw orientation of the vehicle, and the tractor's yaw-orientation is judged by the tractor's yaw orientation. $\Delta \varphi=\varphi_{1}-\varphi_{2}$, is defined as the different value of yaw angle between tractor and trailer.

Table 1. The braking control strategy of the vehicle

\begin{tabular}{ccccc}
\hline Direction of vehicle & Direction of tractor's yaw angle & Relative yaw angle & \multicolumn{2}{c}{ Braking wheel } \\
\cline { 3 - 5 } & & & tractor & trailer \\
\hline Left & $\varphi_{1}>0$ & $\Delta \varphi>0$ & Right steering & Left* \\
Left & $\varphi_{1}>0$ & $\Delta \varphi<0$ & Left steering & Right* \\
Right & $\varphi_{1}<0$ & $\Delta \varphi<0$ & Left steering & Right* \\
Right & $\varphi_{1}<0$ & $\Delta \varphi<0$ & Right steering & Left* \\
\hline
\end{tabular}

'*' indicates the whole wheels of one side of the tractor and the trailer.

The braking force distribution model of differential braking control system is shown in Fig.1. It shows that the differential braking strategy of the vehicle is realized in MATLAB/Simulink. In the model, numeral ' 0 ' is defined as stopping the system, numeral value above ' 0 ' is defined as start the system, and the larger numerical value the larger the brake force.

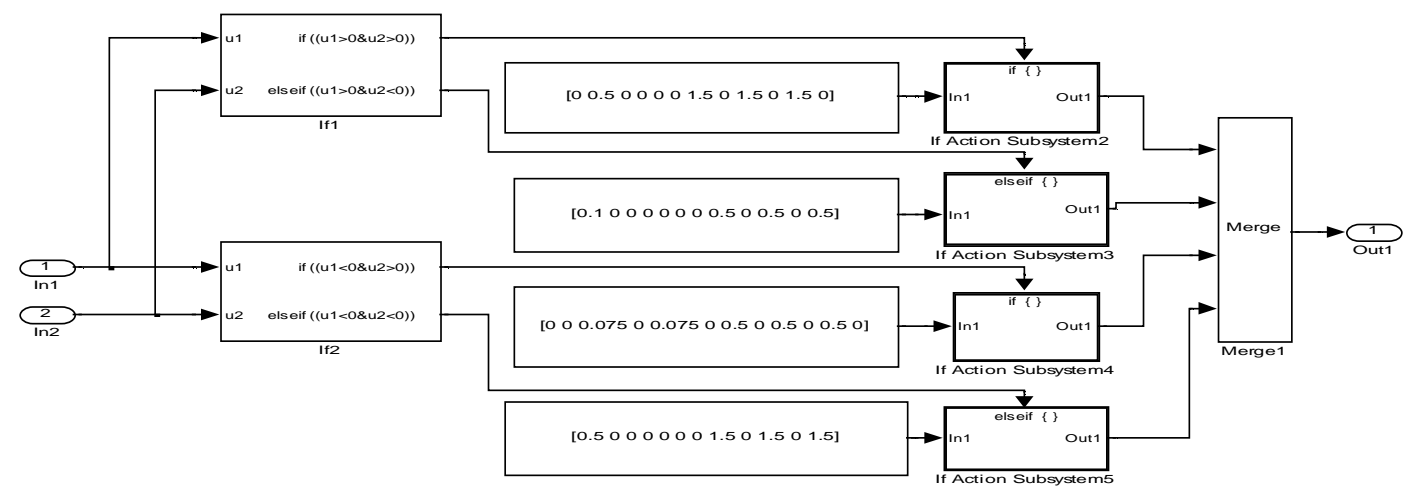

Fig. 1. Braking force distribution model of differential braking control system.

\section{Establishment of Sliding Mode Variable Structure Control System}

According to the need of the control of the high speed shimmy of Tractor-Semitrailer, to establish a sliding mode variable structure control model. The sliding mode variable structure is a control strategy which is not continuous in control. And it has the characters that can make system turning on or turning off in deferent time ${ }^{[6]}$. It's a exceptive, of no fixed structure and nonlinear control. It can force the model following the beforehand settings of 'sliding states' traces moved according to current deviation, state and states' derivative of the system ${ }^{[7]}$.

Based on the sliding mode variable structure control theory, the control model of Tractor-Semitrailer is established. The model's input is the different value of yaw angle between tractor and trailer(being defined as relative yaw angle for short, which is defined in the rest of paper), the variable states are the relative yaw angle and its rate, the output variable is control forces. Through the algorithm of sliding mode variable structure control, the relative yaw angle can be controlled in a certain range, the tractor's yaw angle can be well tracked by the trailer's yaw angle. The established theoretical algorithms of the control system can be written as follows: given a target signal $r$, the error and error rate of the actual signal and the target signal are respectively defined as state variable e and $\delta$, and, 
In Eq. 1 and Eq.2, $r$ means the tractor's yaw angle, $x_{1}$ and $x_{2}$ respectively mean the trailer's yaw angle and its rate, $e$ and $\delta$ respectively mean the relative yaw angle and its rate.

The switching function can be written in Eq.3:

$$
s=c e+£
$$

In Eq.3, $c$ is a constant, here, using the proportional switching control method whose control function can be written in Eq. 3:

$$
u=(\alpha|e|+\beta \mid \oint \operatorname{sgn}(s)
$$

In Eq.4, $\alpha$ and $\beta$ both are constants above zero.

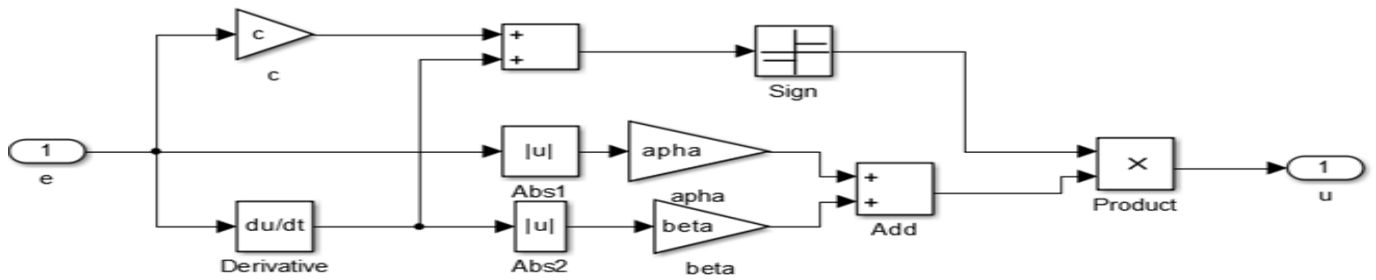

Fig. 2. The control model of sliding mode variable structure.

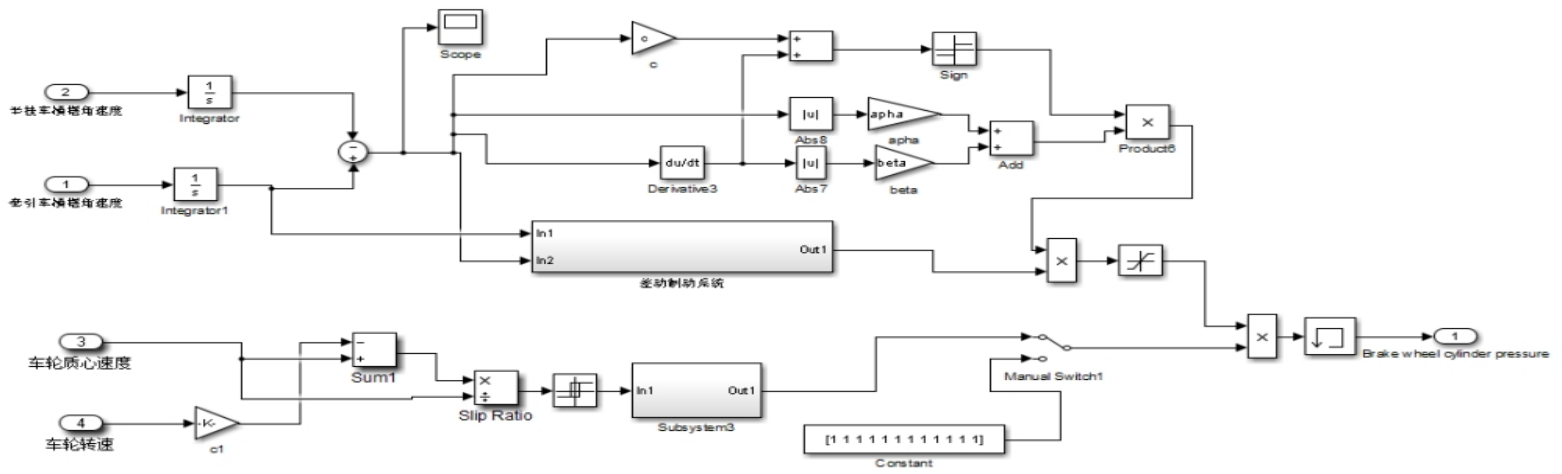

Fig. 3. Differential brake control system model based on sliding mode variable structure control.

Fig. 2 is a sliding mode variable structure control model; Fig. 3 is a model of differential braking control system based on sliding mode variable structure control system, which including the sliding mode variable structure control model (Fig.2), and the braking force distribution model (Fig.1).

A sliding mode variable structure control model based on Eq.1, Eq.2 and Eq.3 is established as shown in Fig. 2. The model is described as follows: the relative yaw angle 'e', as input port, is the result of the integral of tractor's yaw velocity subtracting the integral of trailer's yaw velocity. Both tractor's and trailer's yaw velocities are from vehicle model established in TruckSim(Fig.3). The system output force ' $u$ ' of the output port is used as the braking force to control the wheels of TruckSim vehicle model.

The model in Fig. 3 is described as follows: the brake force which calculated from the model designed in Fig. 2 combines with control strategies of modules of differential brake system designed in Fig. 3, which can carry out the differential brake control to the wheels. The function of the model is to convert the relative yaw angle to output force via the proportional switching control algorithm, and the function is based on the algorithm of sliding mode variable control. The control strategy of differential braking control is used to brake different wheels to obtain the desired yaw moment and reduce the relative yaw angle. 


\section{Simulations and Analyses}

The Simulation model of the control of the high-speed shimmy is shown in Fig.4, including the Simulink controller model and the TruckSim entire vehicle's dynamic model. TruckSim and MATLAB/Simulink simulation methods are as follows: the yaw velocities of tractor's and trailer's center of mass are obtained from the vehicle model in TruckSim. They make integral to obtain their yaw angles and then make subtraction to get the result. Inputting the result to ' $\mathrm{e}$ ' which is the input port of Sliding mode variable control model(Fig.2). After the operation of the model, output force ' $u$ ' of the output system is inputted to the each wheel of TruckSim vehicle model (Fig.4), controlling the braking force of each wheel, more details in Fig. 3 and Fig.4.

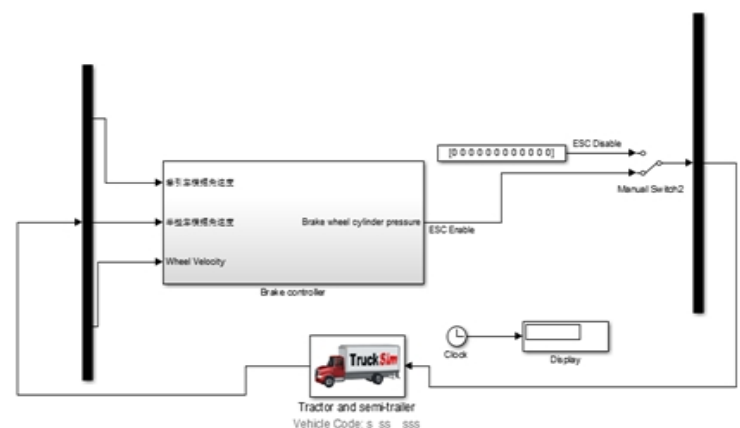

Fig. 4. Simulation model of the control of the high-speed shimmy.

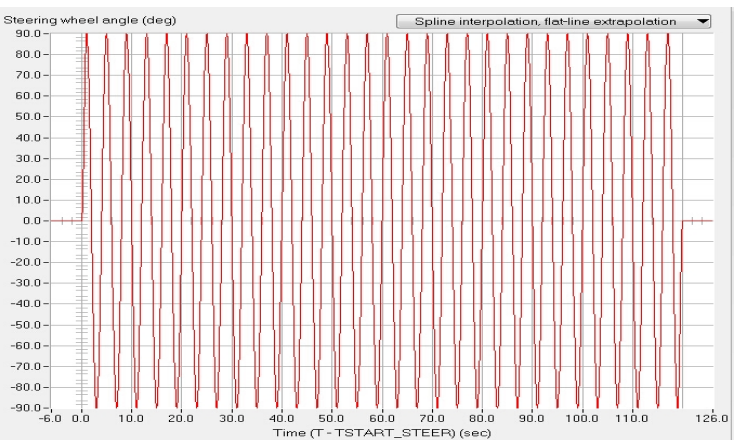

Fig. 5. The steering wheel of inputting the sine of the angle.

In order to better simulate the phenomenon of high speed shimmy, the model of the vehicle is artificially set with a steering wheel sine angle input as shown in Fig.5. Its period is 4 seconds and the maximum amplitude is $90^{\circ}$.

In the simulation experiment, the speed is set $90 \mathrm{~km} / \mathrm{h}$, the simulation time is $30 \mathrm{~s}$. Simulation results of the steering wheel sine angle input mode are shown in Fig. 6 and Fig.7. In the picture,Unit 1 is defined as the center of mass of the tractor, Unit 2 is defined as the center of mass of the trailer, and the meanings of all curves are marked in English in Fig. 6 and Fig.7.

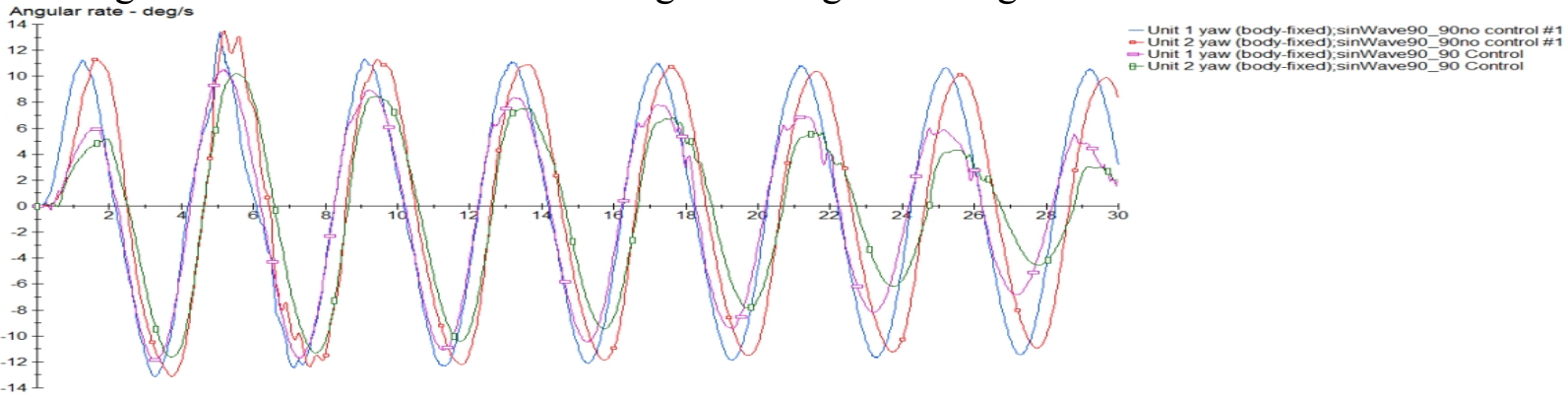

Fig. 6. Comparison of yaw rate of the tractor and trailer before and after control.

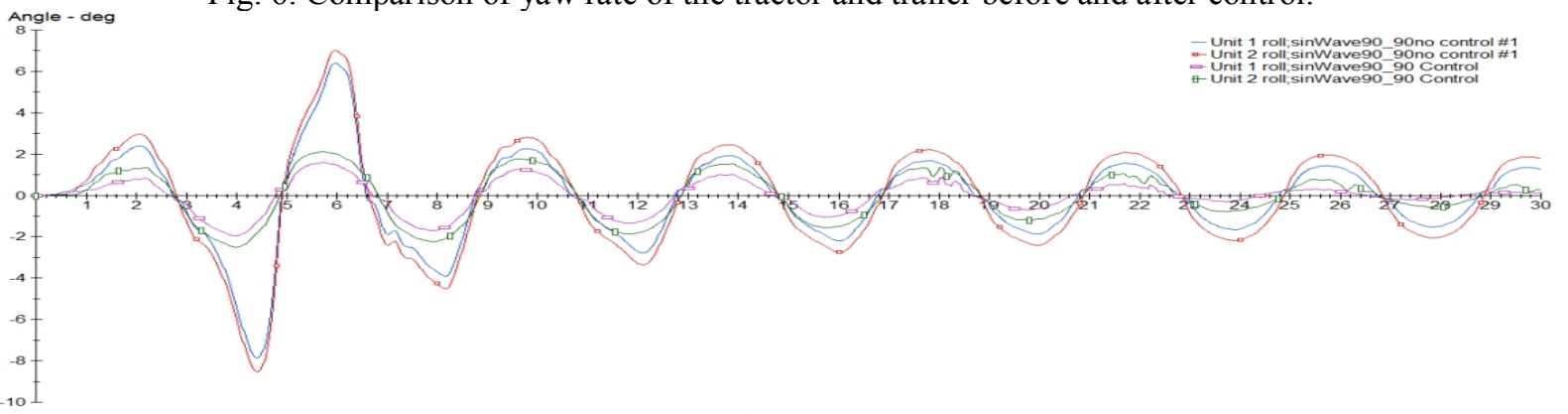

Fig. 7. Comparison of roll angle of the tractor and trailer before and after control.

The comparison between the control front and rear of the tractor and trailer yaw rate is shown in Fig.6. The results shows that when the Tractor-Semitrailer has a high speed shimmy, the peak value of yaw velocity of controlled vehicle is $10 \mathrm{deg} / \mathrm{s}$, which is less than the peak $13.7 \mathrm{deg} / \mathrm{s}$ of the uncontrolled, less $3.7 \mathrm{deg} / \mathrm{s}$, and its amplitude is gradually fading. For the uncontrolled vehicle, its peak value of yaw velocity is relatively large and it makes equal amplitude vibration nearly in 
somewhere $12 \mathrm{deg} / \mathrm{s}$, with no fading. This shows that the designed controller can reduce the yaw rate and can effectively inhibit the high speed shimmy.

The comparison between the control front and rear of the tractor and trailer side dip angle is shown in Fig.7. The results show that when the Tractor-Semitrailer has a high speed shimmy, for controlled vehicle, its side dip angle of shimmy maximum is about $3.3 \mathrm{deg}$. While the uncontrolled, its side dip angle is about $7 \mathrm{deg}$ which is more than doubled compared with $3.3 \mathrm{deg}$, and the amplitude of left and right lateral list is obviously greater than the controlled.Therefore, side dip angle of vehicle without controlled is more easily coming to the rollover threshold and to be dangerous. This shows that under the sliding mode variable structure controlling, it can effectively reduce the side dip angle, improve the lateral stability of Tractor-Semitrailer.

\section{Conclusions}

Through analyzing the causes of Tractor-Semitrailer's high-speed shimmy, we design a controller based sliding mode variable structure and respectively build Simulink controller model and the TruckSim entire vehicle dynamic model. The Simulink control model includes the differential braking control system and the sliding mode variable structure control system.

TruckSim and MATLAB/Simulink simulation methods are used to compare the change curves of the control front and rear yaw rate and the side dip angle of tractor and the trailer.

The results show that the designed controller can reduce the yaw rate and the side dip angle of shimmy in high speed driving, which can effectively inhibit the high speed shimmy and improve the stability of the Tractor-Semitrailer.

\section{Acknowledgments}

The project is supported by Guangxi Natural Science Foundation (NO: 2014GXNSFAA118348) and Educational Commission of Guangxi Province of China (NO: 2013ZD002). The authors are grateful to the science and Technology Department of Guangxi Province and Educational Commission of Guangxi Province for their financial support of this work.

\section{References}

[1]Jinsong Dong, Driving directional stability and brake compatibility control strategy of Tractor-Semitrailer braking in a turn, in Chinese, Chang chun : Jilin University (2010)

[2]Van, D.M. L. M.F.J etal, Analysis of the Lateral Dynamic Behavior of Articulated Commercial Vehicles. Vehicle System Dynamics, 50 ( S1 ) :169-189 (2012)

[3]Xiujian Yang, Yaoping Li and Jian Xiong, Automotive Engineering, 33(6):486-492, In Chinese (2011)

[4]Jun Tian, Research on improved TTR rollover warning and multi-objective stability control algorithm of heavy duty vehicle. In Chinese, Chang chun, China: Jilin University (2011)

[5]Chaoyi, Wei, Study on handling stability of caravan, In Chinese, Zhenjiang, China: Jiangsu University (2008)

[6] Feng You, etal, Journal of Jilin University: Engineering Edition, (5):1296-1302, In Chinese (2014).

[7] Song Chen, Changgao Xia and Xu Sun, Journal of Chongqing Jiaotong University: Natural Science Edition, (4):701-704, In Chinese (2013) 